\title{
¿Converge la fecundidad en Brasil y Argentina? Un enfoque desde las desigualdades
}

\author{
Nicolás Sacco ** \\ Gabriel Borges $^{\star \star \star}$
}

La idea de una tendencia de la fecundidad hacia niveles convergentes en América Latina y el Caribe posee una amplia aceptación, tanto en estudios académicos como así también en los supuestos por detrás de las proyecciones de población de los países de la región. Sin embargo, es poco lo que se sabe sobre los procesos de convergencia demográfica al descomponer la fecundidad según variables de diferenciación social. El objetivo de este artículo fue observar de forma comparada la hipótesis de convergencia en Argentina y Brasil desde 1970 hasta el 2010 de acuerdo a un análisis de periodo de la tasa global de fecundidad (TGF) según regiones estadísticas, niveles educativos y categorías socioocupacionales. Los datos provienen de los censos nacionales de población y las variables fueron armonizadas de manera tal de lograr comparaciones entre ambos países. Se aplicó una modificación de los métodos de la razón $\mathrm{P} / \mathrm{F}$ de Brass y del modelo relacional de Gompertz para estimar los niveles de fecundidad, y se calcularon medidas de convergencia y dispersión para evaluar la hipótesis según las variables de análisis. Los resultados muestran evidencia de que la fecundidad presentó pautas de convergencia durante el período de estudio, especialmente para Brasil, aunque con algunos ciclos de divergencia y diferencias notorias entre los dos países, en particular de aquellos derivados de la posición en la estructura ocupacional.

Palabras clave: Fecundidad. Convergencia demográfica. Estructura social. Argentina. Brasil.

\footnotetext{
* Una versión preliminar de este trabajo fue presentada en el pre-evento "Análisis comparativo de las tendencias demográficas sub-nacionales en América Latina y el Caribe" del Congreso de la Asociación Latinoamericana de Población (Alap) de 2016. Se agradecen los comentarios de los participantes de la mesa y de los revisores anónimos de la revista.

** Centro de Desenvolvimento e Planejamento Regional, Universidade Federal de Minas Gerais (Cedeplar/UFMG), Belo Horizonte-MG, Brasil (nsacco@cedeplar.ufmg.br; http://orcid.org/0000-0003-1078-6142).

*** Instituto Brasileiro de Geografia e Estatística (IBGE) y University of California, Berkeley (gmendesb@hotmail.com; http://orcid.org/0000-0002-8840-328X).
} 


\section{Introducción}

Una de las discusiones más actuales en los estudios de población es sobre si se manifiestan o no pautas de convergencia demográfica entre los países, o entre regiones dentro de cada país, a medida que transcurre el cambio demográfico. Aspecto central para el conocimiento de las causas y consecuencias de las transformaciones sociales, este tema es también pertinente para reflexionar sobre los caminos futuros de la población y sus desigualdades en distintas coyunturas históricas, así como las posibilidades de persistencia de las conocidas asociaciones entre la estructura socioeconómica de las poblaciones y sus características demográficas.

La idea de convergencia demográfica está implícita dentro las teorías de la transición (WILSON, 2001), tanto en mortalidad como en fecundidad. En estudios recientes se ha dado un creciente interés en las distintas metodologías para su operacionalización e interpretación (BORGES, 2016; CASTERLINE, 2001; COLEMAN, 2002; DORIUS, 2008; LANZIERI, 2010; LEE; REHER, 2011; PARRADO; MORGAN, 2008; WILSON, 2001) y el debate teórico sobre estos fenómenos ha generado una gran cantidad de trabajos empíricos, mostrando que los procesos de convergencia no pueden ser explicados por un modelo único.

Para algunos autores, una de las características del proceso histórico de transición demográfica ${ }^{1}$ es que comenzó en una gran pluralidad de contextos, pero hacia el final, sus efectos, en toda latitud, tendieron a converger, tanto en los países precursores de la transición como en aquellos, seguidores o rezagados, del mundo en desarrollo, donde se observan disparidades sociales, económicas y culturales junto a similitudes demográficas (CELADE, 2014; REHER, 2004, 2007). De forma adicional, a pesar de la diversidad de transiciones a nivel global y regional, muy diferenciales, a su vez, dentro cada país, estas ideas fueron sustento conceptual para comprender la dinámica de la población (REHER, 1999) y las regularidades empíricas en las tendencias de la mortalidad y la fecundidad, proporcionando una base teórica para la elaboración de proyecciones de población (WILSON, 2013).

En su análisis a nivel global de la segunda mitad del siglo XX, Wilson (2001) provee resultados sobre hasta dónde el aumento de la esperanza de vida al nacer y la declinación de los niveles de fecundidad convergieron, lo que llevó a desarrollar la idea de un proceso de convergencia global (DORIUS, 2008). En lo que respecta a la fecundidad, especialmente en países desarrollados, una abundante literatura proporcionó evidencia acerca de su convergencia (DORIUS, 2008; LEE; REHER, 2011; REHER, 2004).

Sin embargo, a pesar de estos trabajos, algunos autores sostienen que aún no hay prueba suficiente para respaldar tal hipótesis (DYSON, 2010; KENT; HAUB, 2005; VALLIN;

\footnotetext{
$\overline{1}$ La transición demográfica tiene, al menos, dos significados primarios (JOHNSON-HANKS, 2008). Refiere al cambio histórico específico de altas a bajas tasas de natalidad y mortalidad que muchas poblaciones humanas han experimentado desde mediados del siglo XVIII y, segundo, al conjunto de teorías acerca de las causas y mecanismos detrás de ese cambio -(CALDWELL, 2004; COALE, 1977; DAVIS, 1963; DYSON, 2010; JONES, 1997; KIRK, 1996; LANDRY, 1987; LEE, 2003; LEE; REHER, 2011; MACINNES; PÉREZ DÍAZ, 2009; NOTESTEIN, 1945; REHER, 2004; THOMPSON, 1929), por ejemplo-. En este artículo se distingue entre el cambio empírico y el corpus conceptual acerca de sus causas utilizando el término transición demográfica para aludir a la primera definición y el término teoría de la transición demográfica para indicar la segunda.
} 
MESLÉ, 2004). En el caso de los países en desarrollo, se han encontrado importantes diferencias en el itinerario de la declinación de la fecundidad tanto en los niveles intra-países como entre-regiones (CASTERLINE, 2001). Algunos autores (DORIUS, 2008), estimando los niveles absolutos y relativos de convergencia, han puesto en duda la idea de que en la segunda mitad del siglo XX se dio un periodo de convergencia de la fecundidad: si bien se observa que las tasas de fecundidad a nivel global convergen hacia un mismo nivel en el largo plazo, grandes diferencias se perciben en cada trayectoria particular.

Patarra (1994) concierta en algunos puntos con esos enfoques, al sostener que las tasas demográficas pueden converger a largo plazo, pero argumenta que se trata de un punto discutible en términos de las explicaciones de las transformaciones, que resultan de procesos sociales diferenciales. Dilucidar la forma en cómo interactuarían las dimensiones de la desigualdad social en el proceso de transición de la fecundidad, pone en cuestión la idea de que la convergencia demográfica pueda, o bien coexistir con las desigualdades socioeconómicas (RODRÍGUEZ, 2001) o que, de hecho, dependa de ellas (COLEMAN, 2002).

Mientras que desde un punto de vista global los niveles de fecundidad mostraron una tendencia convergente, de acuerdo a algunos estudios, en América Latina y el Caribe (ALyC), se observaron etapas de divergencia entre 1950-1955 y entre 1980-1985, en sincronía con el inicio y posterior aceleración del proceso cambio de la fecundidad en la región, para luego tomar una fase convergente, desde la década de 1980 hasta principios del siglo XXI (CELADE, 2014, p. 13). En comparación con las regiones menos desarrolladas del mundo, ALyC presenta actualmente la menor dispersión en sus niveles de fecundidad y distintas investigaciones afirman que continuará esta tendencia en el futuro, como parte de un fenómeno global, tanto dentro de cada país como entre grupos sociales y regionales (CELADE, 2014, p. 14).

A pesar del estudio histórico de las tendencias de convergencia/divergencia que se derivan de los distintos tiempos y duración de las etapas de la transición demográfica en cada región, es escasa la literatura existente que indague estas cuestiones en contextos post-transicionales (WILSON, 2013), etapa en la que la mayoría de los países latinoamericanos se encontrará en breve (ONU, 2017). En Europa ya se discute la posibilidad de «destinos demográficos divergentes», con persistentes e importantes diferenciales regionales, aunque con bajos niveles de fecundidad (BILLARI, 2018; KOHLER; ANDERSON, 2016).

En este contexto, cabe preguntarse, ¿cómo han evolucionado los diferenciales de fecundidad según distintas variables de análisis en ALyC? ¿Estamos en presencia de procesos dónde la pertenencia a distintas unidades geográficas, grupos educativos, variable clásica por excelencia utilizada como proxy de la posición social -para esta discusión ver Rodríguez Vignoli (2014) - y estratos socio-económicos no juega el mismo rol convergente al observar la población como un todo? En definitiva, ¿es posible sustentar esta hipótesis de convergencia al incluir en su observación la dimensión de la desigualdad social?

Si bien medida de diversas formas y con distintos indicadores, la noción de convergencia demográfica ha construido una importante literatura y evidencia empírica en una 
gran variedad de coyunturas demográficas. Sin embargo, salvo para la elaboración de proyecciones de población, esta hipótesis, hasta el momento, ha sido escasamente testeada para los países latinoamericanos (BORGES, 2016). En efecto, la mayor parte de los estudios conocidos sobre países de ALyC acerca estos procesos hacen hincapié o bien de forma global o entre regiones dentro de cada país, presentando, sobre todo, evidencia a nivel sub-nacional.

Ante el hecho de que una de las principales discusiones es en qué medida los niveles de fecundidad fueron diferenciales de acuerdo a distintos grupos sociales, regionales, educativos y/o socioeconómicos y cómo fue su evolución durante la transición, resulta llamativo que sean poco conocidos aquellos trabajos que, enfocados en distintas coyunturas, estudien la convergencia diferencial entre sub-grupos poblacionales más allá del agrupamiento de variables regionales o educativas.

Este tipo de perspectiva se hace particularmente necesaria en el caso de los países de $A L y C$, a razón del reconocimiento que la literatura especializada le otorga a la fuerte heterogeneidad de comportamientos demográficos, característica de casi todos los países de la región. En efecto, una amplia corriente de estudios latinoamericana argumenta, fuertemente, que el comportamiento demográfico de las poblaciones (especialmente el relativo a la fecundidad) está visiblemente ligado a las desigualdades sociales y económicas a las que se enfrentan (CAVENAGHI, 2009a; MERRICK; BERQUÓ, 1983; TORRADO, 1993; WOOD; CARVALHO, 1994; ZAVALA DE COSÍO, 1995).

De hecho, para esta línea teórica se concibe a la desigualdad reproductiva como una parte constitutiva de la desigualdad social, uno de los principales problemas de la región (CANALES, 2004; CEPAL, 2014). Esta característica ha sido atribuida a la heterogeneidad estructural de la economía en ALyC, fuertemente diversa en cuanto a niveles de desarrollo, con altos niveles de desigualdad socio-económica, tanto a nivel inter-regional como intra-país según grupos sociales, especialmente aquellos derivados de la posición socio-económica en el mercado de trabajo (CEPAL, 2010).

Esta heterogeneidad se reflejó en la diversidad de patrones de fecundidad, completamente distintos de los observados en la transición en países desarrollados, por ejemplo (CABELLA; CAVENAGHI 2014, p. 7). Además de una caída mucho más rápida, los altos niveles de fecundidad adolescente y las desigualdades internas en los países son peculiaridades específicas de la región (RODRÍGUEZ VIGNOLI; CAVENAGHI, 2014) y el trayecto hacia un régimen de baja fecundidad en ALyC sigue ostentando, aún a fines del siglo XX y principios del XXI, su polarización social característica (CABELLA; PARDO; NATHAN, 2015; NATHAN et al., 2016; ROSERO-BIXBY et al., 2009).

A pesar de relevantes trabajos recientes sobre fecundidad en la región (BINSTOCK; PANTELIDES, 2005; CABELLA; PARDO, 2014; CASTRO, 2017; CAVENAGHI, 2009b; CAVENAGHI; ALVES, 2011; DE MIRANDA-RIBEIRO et al., 2008; NATHAN et al., 2016; ROSERO-BIXBY et al., 2009), en gran medida, estos vacíos de exploración más allá de las tendencias regionales o sub-nacionales suceden por la escasez de datos de calidad, pero 
también por una fragmentaria agenda de investigación sobre el tema y sobre fecundidad en particular (CABELLA; PARDO, 2016). Por estas razones, el objetivo de este artículo fue el de observar las tendencias diferenciales y variaciones de la convergencia de los niveles fecundidad de periodo en ALyC, asumiendo para ello una perspectiva comparativa y de largo plazo, incorporando la descomposición del descenso de la fecundidad en relación a la convergencia territorial y educativa, como así también la relativa a la estratificación social.

Para ello se seleccionaron dos de los países más grandes de la región, Brasil y Argentina, cuyas trayectorias de transición de la fecundidad tuvieron un derrotero, si bien comparable, a la vez muy diferencial y posible de considerar como representativo de otras coyunturas demográficas latinoamericanas. Argentina, junto con Uruguay, representa el modelo de transición temprana en el siglo XX, que ha seguido un curso gradual de la caída de la fecundidad. Ese modelo comprende, inclusive, periodos de aumento de la fecundidad a lo largo de la transición. Se suma a eso un patrón de fecundidad tardío, que sitúa ese modelo más cerca de la experiencia de los países de Europa Occidental que de los demás países latinoamericanos (CHACKIEL, 2004b). Por otro lado, Brasil ha tenido una fecundidad más jóven y una transición de la fecundidad mucho más rápida, representando un modelo clásico en América Latina, que es compartida por distintos países de la región, aunque en periodos distintos. En otras palabras, el proceso de descenso de la fecundidad (y la evidencia acerca de su homogeneidad) se dio de forma muy diferencial en Argentina y Brasil, característica que perfila un escenario privilegiado para testear la hipótesis de convergencia entre países, intra-países y entre grupos socio-económicos.

Este recorte resulta también relevante ya que la trayectoria de estos dos países, caracterizados por abarcar algunas de las zonas más desarrolladas de la región, fue muy diferente al de otros países similares en otras regiones del mundo desarrolladas y dentro mismo de ALyC, dando la posibilidad de re-abordar la discusión acerca de la idea de convergencia global.

Específicamente, se realizó una observación transversal, en clave descriptiva y comparativa, de la Tasa Global de Fecundidad de periodo (TGF) de estos dos países, según cada región (estadística), niveles educativos y categorías socio-ocupaciones, a partir de la utilización de técnicas demográficas indirectas, empleando las bases de los censos de población de 1970 a 2010.

Los términos "convergencia" y "divergencia” han sido frecuentemente utilizados sin una conceptualización clara, lo que puede llevar a interpretaciones distintas, o incluso contradictorias. En este trabajo, se formalizó este concepto a través de dos medidas estadísticas.

Siguiendo tres preguntas de trabajo, a saber: ¿ha disminuido la variación de la fecundidad entre distintas regiones, niveles educativos y grupos socio-ocupacionales?; ¿qué evidencia existe de convergencia demográfica? y, ¿cuáles son las diferencias entre Argentina y Brasil al respecto?, se elaboró la siguiente hipótesis: en el curso de la transición de la fecundidad, la convergencia de la TGF no siempre es verificable, relación que se hace perceptible al considerar atributos relativos a la diferenciación social, en particular, la estratificación socio-ocupacional. 
A pesar de que la heterogeneidad en el calendario y la convergencia en la intensidad de los comportamientos reproductivos pueden coincidir dentro de una misma coyuntura demográfica (CABELLA; PARDO, 2016; CAVENAGHI; ALVES, 2011), se optó por focalizar el análisis en el nivel de la fecundidad a partir de una medida de periodo, la TGF, y no de otros indicadores como la distribución de la paridez y/o el calendario, abordados en otros trabajos -véase al respecto, por ejemplo, Nathan y Pardo (2016)- porque las desigualdades de la TGF tienden a reflejar también, en cierta medida, los diferenciales en la paridez y calendario. Además, históricamente la desigualdad reproductiva más estudiada ha sido la relativa a la intensidad: la diferencia en el número de hijos tenidos por las mujeres en distintos grupos sociales, socio-ocupacionales, económicos, étnicos o religiosos fue la faceta más conocida de los diferenciales relativos a esta dimensión demográfica (CEPAL, 2006).

Para los objetivos planteados por este artículo, se optó estudiar la convergencia de periodo más que la de cohorte. Se decidió dejar para futuros trabajos un análisis de la convergencia de la fecundidad a través del uso del total de hijos nacidos vivos, dado que se consideró que la interpretación de convergencia de cohorte estaría mostrando la convergencia entre cohortes de nacimiento, mientras que la unidad de análisis del artículo no son las cohortes sino las características del descenso de la fecundidad de momento y el supuesto de esa caída a futuro (supuesto presente, a su vez, en la elaboración de proyecciones de población de los países seleccionados).

El punto de vista de periodo para el análisis de la convergencia de la fecundidad, a pesar de algunos de los problemas señalados, por ejemplo, por Parrado (2016), posee sin embargo la ventaja de resumir las condiciones sociales y económicas del momento. En contextos de caída de la fecundidad se espera que los grupos que contribuyen a esa caída mantengan a futuro bajos niveles. En el análisis de la paridez media al final del periodo reproductivo, más allá de los problemas particulares de estos datos, no sólo la unidad de análisis recaería en cohortes particulares, sino que las condiciones que muestran los resultados condicionan el análisis a cohortes particulares (y por lo tanto a períodos históricos limitados).

De forma adicional, la TGF de periodo (a diferencia de otros indicadores más refinados) resulta una medida que aún muestra una gran capacidad explicativa dado el particular contexto de transición de la fecundidad de ALyC, muy diferencial al de los países que experimentaron procesos hacia la postergación de los nacimientos (CABELLA; PARDO, 2016; CAVENAGHI; ALVES, 2016).

Dado los objetivos planteados, adicionalmente se evalúa, en consecuencia, si los supuestos detrás de las teorías demográficas y proyecciones se observan (o no), en lo que respecta a las pautas de convergencia demográfica. En efecto, las expectativas con relación al nivel de fecundidad juegan un rol predominante en la selección de niveles límite de fecundidad en las proyecciones de población de la región. En la preparación de proyecciones sub-nacionales la mayor parte de los demógrafos utilizan el método por componentes, que requiere de fuertes supuestos (de periodo) acerca de la trayectoria futura de niveles 
de migración, mortalidad y fecundidad (CAVENAGHI, 2012). De hecho, la convergencia en los niveles fecundidad está implícita en las proyecciones oficiales de Brasil del Instituto Brasileiro de Geografia e Estatística (CAMPOS; BORGES, 2015; IBGE, 2013) y Argentina, del Instituto Nacional de Estadística y Censos (INDEC, 2013).

En lo que sigue del artículo se aborda, en primer lugar, el contexto y los antecedentes que se conocen sobre la fecundidad y su convergencia en los países seleccionados. Luego se describen las características de los datos censales utilizados y las principales herramientas indirectas de análisis. En Resultados se presentan i) las diferencias de la TGF a nivel del total de cada país por períodos y las diferencias inter-regionales y ii) los diferenciales según niveles educativos y categorías socio-ocupaciones. Por último, se discuten los resultados encontrados en la sección Conclusiones.

\section{Trayectorias diferenciales de la fecundidad}

Teniendo en cuenta sobre todo lo sucedido luego de la segunda mitad del siglo XX, ALyC transitó una rápida caída de su fecundidad, si bien de forma comparable, poco homogénea entre los países de la región (CHACKIEL, 2004a, 2004b; CHACKIEL; SCHKOLNIK, 2004; GUZMÁN et al., 2005; GUZMÁN et al., 1996; SCHKOLNIK; CHACKIEL, 2004; ZAVALA DE COSÍO, 1992). El vínculo entre la desigualdad estructural y las diferentes dinámicas demográficas tanto a nivel regional como intra-países, son peculiaridades bien estudiadas por la literatura. Diversos estudios (CEPAL, 2011; CHACKIEL; SCHKOLNIK, 2004; GUZMÁN, 1991; GUZMÁN et al., 1996; ITABORAÍ, 2015; TORRADO, 2007; WOOD; CARVALHO, 1994) argumentan acerca de la coexistencia de dinámicas diferenciales, de acuerdo a la cual los países con menor desarrollo relativo (y las mujeres de clases bajas) ostentan una fecundidad más alta y más temprana que la de los países más desarrollados (y de las clases medias y altas), particularidad compartida con el resto de los países de fecundidad intermedia en otras regiones (ONU, 2002).

En ALyC, a partir de la década de 1960, luego de una larga etapa distinguible por la relativa estabilidad de la TGF en niveles altos, se observó una veloz reducción hasta los años noventa, cuando se dio una fase de descenso más lento pero sostenido, tendiente hacia niveles cercanos al reemplazo poblacional. En los últimos 25 años el nivel de la fecundidad se acerca a un régimen de baja fecundidad, proceso que se inició en la década de 1970, caracterizado por una creciente homogeneización en torno al promedio de la región (CABELLA; PARDO, 2014).

En el escenario escogido para revisitar la hipótesis de convergencia, los países seleccionados representan casos muy particulares de este proceso. En efecto, el derrotero de la fecundidad se dio prácticamente de forma asemejable en todos los países de ALyC, a exepción de Argentina y Uruguay (CHESNAIS, 1992).

En Argentina, la reducción temprana de la fecundidad ha sido un rasgo distintivo de su transición demográfica (PANTELIDES, 1989, 2006). A pesar del fuerte descenso observado 
de sus niveles entre fines del siglo XIX y principios del siglo XX, una importante inflexión se dio en el cierre del siglo XX. A fines de la década de 1970, la desaceleración de la caída de la fecundidad, en un contexto de aumento de las heterogeneidades sociales y económicas, revelaba dinámicas demográficas diferenciales según la posición socioeconómica y/o geográfica (GOVEA BASCH, 2013).

Los estudios especializados acuerdan al interpretar este proceso de cambio fue fuertemente desigual, en donde, todavía a fines del siglo XX, en algunas regiones y grupos sociales, era muy incipiente la transición, reconociendo la existencia de diversos diferenciales en su recorrido (BINSTOCK; CERRUTI, 2016; BINSTOCK; PANTELIDES, 2005; GIUSTI, 1993; LÓPEZ, 2006; LÓPEZ; MARIO, 2009; MAZZEO, 1995; OTERO, 2004; PANTELIDES, 1997; TORRADO, 2003).

Si bien se conocen distintos trabajos que aborden la peculiar transición de la fecundidad en ese país y sus tendencias generales, son poco conocidos aquellos que remitan específicamente a las pautas de convergencia. Grushka (2014, p. 74) observó un proceso convergente en la TGF durante el periodo 1980-2010, “que se explica, en parte, por estar acercándose a límites supuestamente asintóticos y por el fenómeno conocido como regresión a la media: las jurisdicciones con mayores aumentos/disminuciones son las que parten de niveles más reducidos/elevados".

A diferencia de Argentina, la transición de la fecundidad en Brasil representa, a grandes rasgos, un prototipo de lo experimentado por el promedio de la región: una relativamente alta TGF se mantuvo en niveles prácticamente constantes entre 1940 y 1960 alrededor de 6 hijos por mujer ${ }^{2}$ (CARVALHO; WONG, 1992, p. 114-5)o y a mediados de la década de 1960 comienza su descenso a nivel del total del país. El descenso de los niveles de fecundidad decididamente rápido se inicia un poco antes de entrar a la década de los años '70 (FERNANDEZ; CARVALHO, 1986; GOLDANI, 2002; MARTINE, 1996). En efecto, en esos años se observó una acelerada caída, primero en las regiones relativamente más desarrolladas, diseminado luego en los años '70/'80 en distintos grupos sociales y regionales, pasando de una de TGF de 5.8 para 1970, 4.3 para 1980 (CARVALHO; WONG, 1992, p. 115), alrededor de 2.5 hacia 1990 (ADSERA; MENENDEZ, 2011) llegando a niveles por debajo del nivel de remplazo en 2010 (ZANATTA COUTINHO, 2016).

Este descenso alcanzó niveles inesperadamente bajos, si bien con persistentes diferencias territoriales (CAMARANO et al., 1999), tendientes, según algunos estudios, a uniformar las regiones alrededor del promedio nacional, incitando la convergencia de todos los grupos sociales a tamaños de familia cada vez más reducidos (CARVALHO, 1997; CARVALHO; WONG, 1992). Este hecho fue asociado a una homogeneización de los niveles de fecundidad a consecuencia de los efectos de la modernización, por ejemplo, como efecto de los medios de comunicación de masas (FARIA, 1989), más que a un proceso de difusión e interacción social donde seguidores adoptarían comportamientos de líderes (POTTER et

\footnotetext{
$\overline{2}$ Rios-Neto (2000) indica también una caída previa de la fecundidad entre el final del siglo XIX y los años 1940.
} 
al., 2010). Estos cambios no se restringieron solamente al nivel, sino que se observaron también variaciones en la edad media de la fecundidad y en la composición de la fecundidad por orden de nacimientos (DE MIRANDA-RIBEIRO et al., 2016, p. 32).

En contraste con otros países rezagados en el proceso de transición demográfica, la rápida declinación de la fecundidad en Brasil - cuyas causas aún continúan siendo fuente de debate- fue, para algunos autores (BAJRAJ et al., 1997; CAMARANO et al., 1999; CARVALHO; WONG, 1992; DINIZ ALVES, 2008; FERNANDEZ; CARVALHO, 1986; GOLDANI, 2001, 2002; RODRIGUEZ WONG, 1983), no homogénea entre las regiones, e incluso a principios del siglo XX sus niveles continúan siendo variables a lo largo del país. Estudios previos sugieren que la evidencia empírica es insuficiente para demostrar la hipótesis de estabilización de las tasas fecundidad en un nivel homogéneo (CAMARANO, 1996a, 1996b, p. 19; CAMARANO et al., 1999), siendo los impactos de las variables socioeconómicas (BERQUÓ; CAVENAGHI, 2004, p. 17; CAVENAGHI; ALVES, 2011) y educativas (ADSERA; MENENDEZ, 2011; DE MELLO MOREIRA; FUSCO; FRIAS; OLIVEIRA, 1991; MERRICK; BERQUÓ, 1983; RIOS-NETO; DE MEIRELES GUIMARÃES; ZANATTA COUTINHO, 2016) aún importantes a la hora de explicar las diferencias encontradas entre las regiones.

\section{Datos y métodos}

Dado que las estadísticas vitales no cubren la totalidad de información de nacimientos con información amplia y de calidad (tanto en los países seleccionados como en el resto de los países de $A L y C)$, al adoptar una perspectiva de larga duración que incorpora tanto la descomposición del descenso de la fecundidad por estratos socio-ocupacionales como la convergencia territorial y educativa, los datos censales, de forma prácticamente ineludible, son la única fuente que permite abordar la perspectiva de análisis propuesta, dado que, a diferencia de las estadísticas vitales, admiten cruces con atributos relativos a la diferenciación social.

Se utilizaron las bases de datos de los censos nacionales de población de los países seleccionados de 1970 a 2010, en el formato de microdatos (IPUMS-Internacional ${ }^{3}$ ) como aquellos provistos por las oficinas nacionales de estadística para los censos más actuales. No sólo la disponibilidad primó para elegir las bases seleccionadas, sino también su posibilidad de homologación, tanto entre los censos de cada país como entre países, tarea que se llevó a cabo gracias a la riqueza de IPUMS-I que ya incluye variables armonizadas por país. ${ }^{4}$ Para calcular la TGF se utilizaron los datos de hijos nacidos vivos el último año, la paridez acumulada y el total de mujeres en edades reproductivas (15-49 años).

\footnotetext{
${ }^{3}$ Minnesota Population Center. Integrated Public Use Microdata Series, International: Version 6.4 [Machine-readable database]. Minneapolis: University of Minnesota, 2015. A partir de ahora se cita esta fuente como IPUMS-I.

${ }^{4}$ El censo de 1970 de Argentina y el de 1960 de Brasil fueron excluidos del análisis ya que en ambos no fueron relevados el total de hijos nacidos vivos el último año.
} 
La tendencia a la subdeclaración del número de hijos el último año es ampliamente conocida. Diversos métodos demográficos han sido propuestos para hacer frente a esta cuestión, como el método P/F de Brass $(1964,1968,1975)$ y el modelo relacional de Gompertz (BRASS, 1978; ZABA, 1981), entre los más utilizados. El método P/F de Brass es la técnica de estimación indirecta más usada para calcular la fecundidad (MOULTRIE; DORRINGTON, 2008) y consiste básicamente en relacionar la fecundidad reciente con la paridez acumulada, proporcionando factores de ajuste para evaluación y futura corrección de la fecundidad en el último año. La idea es que, bajo ciertos supuestos, como fecundidad constante, la fecundidad acumulada de una cohorte en una cierta edad, por ejemplo $\mathrm{P}(20-24)$, sería igual a la fecundidad reciente acumulada hasta esa edad, F(20-24). La razón entre estos dos factores sería usada para corregir la fecundidad reciente. Diversos trabajos han destacado limitaciones del método $\mathrm{P} / \mathrm{F}$ de Brass, especialmente en contextos de rápido descenso y cambio de la estructura etaria de la fecundidad (CARVALHO et al., 2016; CASTANHEIRA; KOHLER, 2015; MOULTRIE; DORRINGTON, 2008; SCHMERTMANN et al., 2013).

El método relacional de Gompertz es un refinamiento del método P/F de Brass, que parte del mismo principio de comparar la fecundidad reciente con la paridez acumulada, pero usando un modelo relacional capaz de capturar la paridez y fecundidad acumulada por edad. El método relaja la suposición de que la fecundidad permanece constante en el pasado. Como modelo relacional, el método estima la fecundidad a través de la modificación de un calendario de fecundidad standard. Las informaciones derivadas de la paridez (puntos $P$ ) y las que provienen de las tasas específicas de fecundidad (puntos $F$ ) deben ser consistentes. Además de la ventaja de no requerir fecundidad constante en el pasado, los resultados de la aplicación de este método a través del análisis de las tendencias de los puntos P y F por edad permiten la evaluación de los supuestos (MOULTRIE, 2013b).

Berquó y Cavenaghi (2014) señalaron dos limitaciones del método relacional de Gompertz. La primera, es que el método cambia significativamente la estructura por edad de la fecundidad. Esta es una limitación particularmente importante para el contexto de este trabajo, ya que es reconocido que la información por edad es de buena calidad en Brasil (CARVALHO et al., 2016; CAVENAGHI; ALVES, 2016) y Argentina (SACCO, 2015), aunque subdeclaradas. Asumir que el error de declaración del número de hijos nacidos vivos el último año es aproximadamente constante por edad, es uno de los principales supuestos del método $\mathrm{P} / \mathrm{F}$ de Brass.

La segunda limitación del método relacional de Gompertz es que el ajuste final es muy dependiente de un análisis visual, que puede ser laborioso si se compara al uso de la razón P/F de Brass (BERQUÓ; CAVENAGHI, 2014).

Basado en estas consideraciones y en el contexto de calidad de las informaciones y características de la fecundidad en Brasil y Argentina, este trabajo propone una modificación en la aplicación de los métodos de la razón P/F de Brass y del modelo relacional de Gompertz, utilizando sus principales ventajas, al mismo tiempo que hace frente a sus principales limitaciones. 
Asumiendo que la fecundidad reciente declarada es de buena calidad, excepto por un factor constante por edad, es posible calcular sólo un factor de ajuste (P/F) a través del método relacional de Gompertz, en lugar de reestimar la fecundidad cambiando la estructura etaria. Este cambio supera una de las limitaciones del método relacional de Gompertz, al mismo tiempo que relaja la hipótesis de fecundidad constante en el pasado.

Para resolver la segunda limitación, se propone un criterio único para la elección de los puntos $\mathrm{P}$ y $\mathrm{F}$ y grupos etarios para el ajuste de la fecundidad reciente, basado en la literatura y en el conocimiento acumulado sobre los patrones de error de declaración de la fecundidad reciente y paridez acumulada. La elección de los grupos utiliza las mismas suposiciones del método P/F de Brass en relación a la naturaleza de los errores de declaración: la información sobre fecundidad reciente es subestimada e independiente de la edad; la información de la paridez acumulada posee errores de omisión crecientes con la edad. Moultrie (2013a) sugiere que se eliminen los puntos $P$ en preferencia a los puntos $F$, ya que la paridez acumulada está, usualmente, más sujeta a errores que la fecundidad reciente. Además, puntos $\mathrm{P}$ y F en edades avanzadas no deben ser usados, porque tienden a presentar menor consistencia entre las dos informaciones. Moultrie (2013a) también propone que se elimine la información de las mujeres con menos de 20 años, debido al pequeño número de eventos en estas edades.

Basado en estas recomendaciones, se utilizó la información de fecundidad reciente de las mujeres de 15 a 44 años y la paridez acumulada de las mujeres de 15 a 29 años. Se optó por incluir las mujeres de 15 a 19 años porque en el contexto Latinoamericano este grupo ha tenido importancia en la determinación de las tendencias de fecundidad, con niveles proporcionalmente mucho más altos que en otras regiones (DI CESARE, 2007; RODRÍGUEZ, 2017; RODRÍGUEZ VIGNOLI; CAVENAGHI, 2014).

Una vez calculada la fecundidad reciente ajustada por el modelo de Gompertz relacional, es posible calcular las razones $\mathrm{P} / \mathrm{F}$. Se utilizó en este trabajo el promedio de las razones P2/F2 y P3/F3, referentes a los grupos etarios 20-24 y 25-29. Los criterios descritos anteriormente se mostraron adecuados para el análisis de las 116 dimensiones analizadas en este trabajo, con alta consistencia entre las informaciones derivadas de la paridez (puntos P) y las que provienen de las tasas específicas de fecundidad (puntos F). ${ }^{5}$ Comparaciones con fuentes de datos alternativas, por ejemplo del RIPSA (2012), muestran que los resultados producidos por técnica de Gompertz modificada parecen más consistente que los resultantes de la aplicación de la técnica $\mathrm{P} / \mathrm{F}$ de Brass.

Con objetivo de controlar la consistencia interna de la información utilizada, además del chequeo con las estimaciones oficiales del INDEC y del IBGE, fue calculada la razón de niños-mujeres (el número de niños de 0-4 años por mujeres de 15 a 49 años de edad de población) a nivel regional, los cuales mostraron una relación lineal con los indicadores de fecundidad calculados por el método propuesto en cada nivel de análisis.

\footnotetext{
${ }^{5}$ La única excepción fueron mujeres de nivel educativo alto. Estos resultados sugieren un efecto cohorte entre las mujeres, sobre todo en el caso de Brasil, por las importantes variaciones en los niveles educativos a lo largo del periodo de estudio.
} 
Una vez computadas las TGF para cada región, nivel educativo y categoría socio-ocupacional, fue también calculada una medida de dispersión (el coeficiente de variación o CV) para medir lo que es comúnmente llamado convergencia $\sigma$. El CV fue elegido por ser una medida de dispersión relativa que es recomendada para el análisis de la fecundidad, una vez que una diferencia de 0,5 niños tiene una importancia menor en altos niveles de fecundidad que en niveles cerca del reemplazo (BORGES, 2016). Por esa misma razón, los gráficos de la TGF serán mostrados en escala logarítmica.

Además, fue calculada la convergencia $\beta$ (SALA-I-MARTIN; CENTRE FOR ECONOMIC POLICY, 1995), que indica en qué medida la variación de la fecundidad es distinta en relación a los niveles iniciales, y es dada mediante la fórmula:

$$
\ln \left(Y_{i, t} / Y_{i, 0}\right) / t=\alpha+\beta \ln \left(Y_{i, 0}\right)+\varepsilon_{i}
$$

donde $Y_{i, t}$ es el indicador a ser medido para la región $i$ el tiempo $t$ y $\beta$ es el coeficiente de convergencia. Un valor negativo en el coeficiente de convergencia $\beta$ indica convergencia, mientras que valores positivos del coeficiente muestran divergencia (DORIUS, 2008).

Las regiones (delimitadas de forma comparable en el tiempo) fueron construidas mediante el agregado de provincias según los criterios estadísticos de cada país. A su vez, los niveles educativos fueron agrupados según: nivel bajo (menos de primaria completa); nivel medio (primaria completa); nivel alto (secundaria completa y más). Las categorías socio-ocupacionales fueron consideradas de forma tal que permitieron comparaciones entre los países bajo análisis. Para ello se construyeron series homogeneizadas según la Clasificación Internacional Uniforme de Ocupaciones (CIUO) (OIT, 2010) en su versión 1988 a nivel de 1 dígito 6 y se reagruparon sus 9 categorías en 5 estratos sociales (lo que permitió mayor flexibilidad para el análisis de los datos), de acuerdo al Cuadro 1.

Para el caso de la observación de la TGF según categorías socio-ocupacionales, las mujeres fueron clasificadas de acuerdo a la pertenencia a la categoría socio-ocupacional del jefe/a o persona responsable por el hogar tal como propone Torrado (1992), que permite, además, realizar comparaciones con estudios que siguen una estrategia similar al considerar la clase social familiar - por ejemplo Itaboraí (2016).

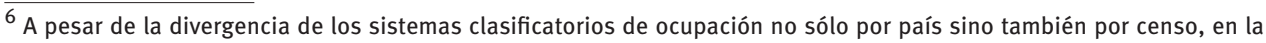
mayoría de los casos esta información está incluida en las bases del IPUMS-I, pero en algunos censos, como en el caso de la Argentina en 1991 y 2001, fueron reconstruidas de forma tal de lograr series lo más compatibles posibles a mayor nivel de agregación, siguiendo lo propuesto por Sacco y Riveiro (2016).
} 
CUADRO 1

Esquema de condición socio-ocupacional

\begin{tabular}{|c|c|c|c|}
\hline CIUO-88 & Categorías socio-ocupacionales & Sigla & Estrato social \\
\hline $\begin{array}{l}\text { Grupo 1: Miembros del poder ejecutivo y de } \\
\text { los cuerpos legislativos y personal directivo } \\
\text { de la administración pública y de empresas }\end{array}$ & $\begin{array}{l}\text { Empresarios, directores de empresas } \\
\text { y funcionarios públicos superiores }\end{array}$ & DIREC & \multirow{3}{*}{$\begin{array}{l}\text { Alto y } \\
\text { medio }\end{array}$} \\
\hline $\begin{array}{l}\text { Grupo 2: Profesionales científicos e } \\
\text { intelectuales }\end{array}$ & \multirow{2}{*}{ - Profesionales y técnicos } & \multirow{2}{*}{ PROF } & \\
\hline $\begin{array}{l}\text { Grupo 3: Técnicos y profesionales de nivel } \\
\text { medio }\end{array}$ & & & \\
\hline Grupo 4: Empleados de oficina & \multirow{2}{*}{$\begin{array}{l}\text { Empleados, trabajadores y } \\
\text { vendedores de los servicios }\end{array}$} & \multirow[b]{2}{*}{ EMPL } & \multirow[b]{2}{*}{ Empleados } \\
\hline $\begin{array}{l}\text { Grupo 5: Trabajadores de los servicios y } \\
\text { vendedores de comercios y mercados }\end{array}$ & & & \\
\hline $\begin{array}{l}\text { Grupo 6: Agricultores y trabajadores } \\
\text { calificados agropecuarios y pesqueros }\end{array}$ & $\begin{array}{l}\text { Agricultores y trabajadores } \\
\text { calificados de actividades primarias }\end{array}$ & AGRI & Agricultores \\
\hline $\begin{array}{l}\text { Grupo 7: Oficiales, operarios y artesanos de } \\
\text { artes mecánicas y de otros oficios }\end{array}$ & \multirow{2}{*}{$\begin{array}{l}\text { Oficiales y trabajadores de } \\
\text { actividades industriales }\end{array}$} & \multirow{2}{*}{ TRAB } & \multirow{2}{*}{ Trabajadores } \\
\hline $\begin{array}{l}\text { Grupo 8: Operadores de instalaciones y } \\
\text { máquinas y montadores }\end{array}$ & & & \\
\hline Grupo 9: Trabajadores no calificados & Trabajadores no calificados & NCAL & \multirow{2}{*}{ - Bajo } \\
\hline Sin especificar & Sin especificar & SESP & \\
\hline
\end{tabular}

* Las fuerzas armadas y los desocupados (para aquellos casos en donde no les fue relevada la última ocupación) fueron incluidos en este nomenclador según nivel educativo: alto en DIREC, medio en PROF, bajo en TRAB y desconocido en NCAL.

Nota: se excluyen los trabajadores inactivos.

\section{Resultados}

\section{Tendencias de la TGF según las variables analizadas}

Las tendencias de la TGF a lo largo del período de observación (Gráfico 1) se muestran para Argentina marcadamente diferenciales por región. En el Norte (Nordeste y Noroeste) se presentan sus valores más altos, superando los 4,5 hijos por mujer en 1980. Estas dos regiones se mantienen al mismo tiempo como las de mayor nivel de TGF hacia 2010. La tasa más baja fue observada en el Gran Buenos Aires y la región Pampeana, oscilando alrededor de 3 hacia 1980. También son estás las regiones las que para el 2010 se mantienen por debajo del resto. A excepción del cambio de posición entre las regiones de Cuyo y Patagonia en la última década, el orden de la TGF entre las regiones se mantiene durante todo el periodo analizado. Si bien las regiones que ya mantenían los menores niveles de TGF continúan una tendencia descendente a lo largo del período de observación, el declive es un poco más pronunciado en las regiones que hacia 1980 ostentaban los más altos niveles de fecundidad.

Comparando iguales etapas (a partir de 1980), Brasil se caracteriza por un nivel general más alto de la TGF, en el promedio de todas sus regiones. Pero, a su vez, a lo largo del periodo de estudio se distingue un descenso mucho más pronunciado que el de Argentina, superando los niveles más bajos de fecundidad de este país hacia el 2010, en todas sus regiones (salvo en la región Norte). Este proceso comienza en los años noventa, pero ya 
durante el lapso 2000-2010 se da con mayor claridad. La caída de los niveles de fecundidad en los últimos 40 años es consistente en todas las regiones y el ranking regional de la TGF se mantiene inalterado, aunque la velocidad de caída sea distinta dependiendo del periodo y de la región analizada.

\section{GRÁFICO 1}

Tasa global de fecundidad, según región (escala logarítmica) Argentina y Brasil - 1970-2010
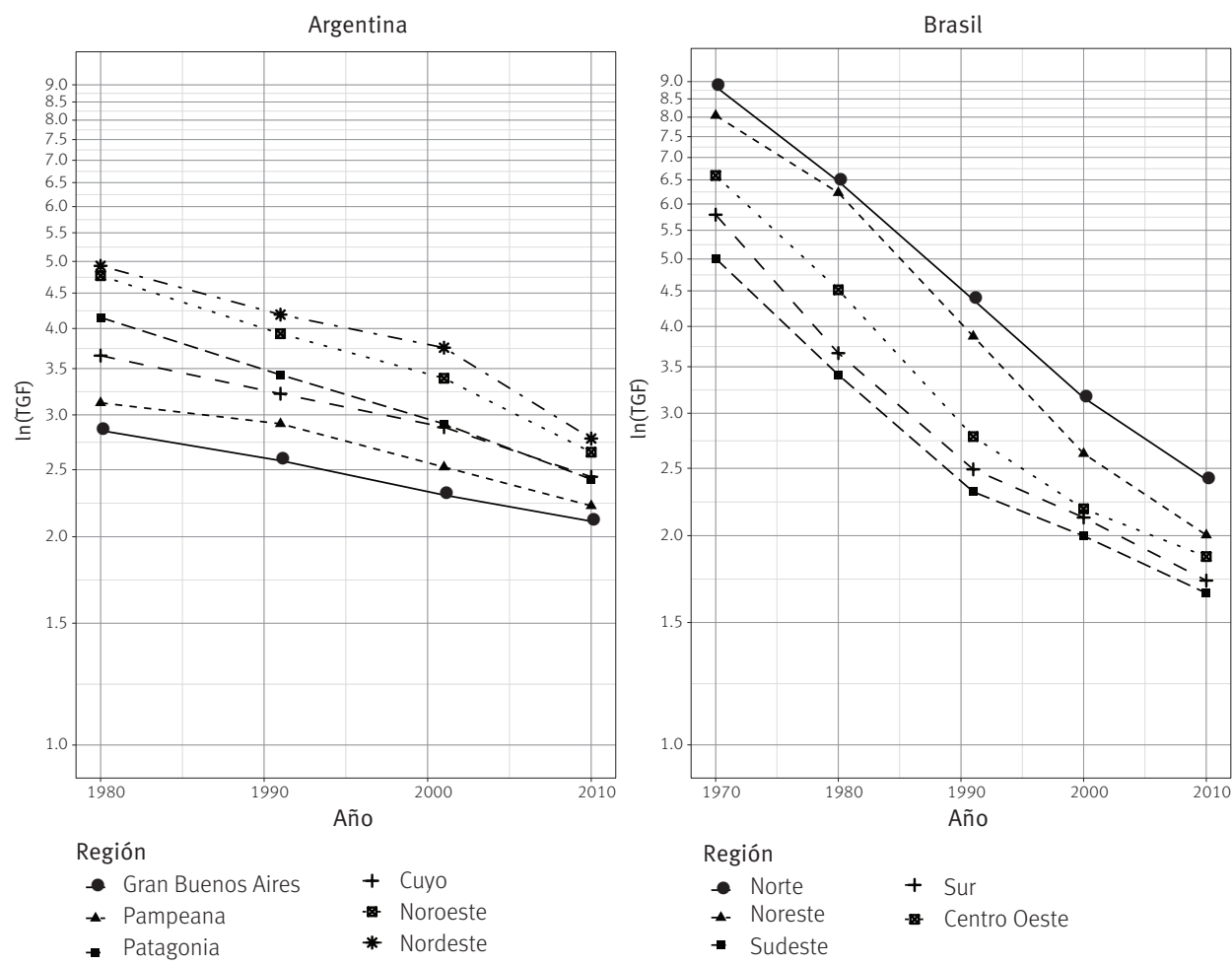

Fuente: Elaboración propia en base a IPUMS-I e INDEC.

Al considerar la fecundidad de los dos países bajo análisis según sus niveles educativos se observa (Gráfico 2) que la TGF en Argentina se mantiene en valores cercanos a 5 hijos por mujer en el nivel educativo bajo, hasta el período 2001-2010, cuando moderadamente es descendiente a 4,2. El nivel educativo medio muestra para el lapso 1980-2001 tendencias ascendentes hasta 2001-2010, cuando alcanza un valor alrededor de 3,5. A diferencia de estos dos niveles, el nivel educativo alto mantiene una clara y sostenida declinación, pasando una TGF de 2,2 en 1980 a 1,5 en 2010, obteniendo valores por debajo del nivel de reemplazo en 1991. A nivel general las tendencias de la TGF son, de cualquier manera, descendientes, dado el peso creciente de los niveles educativos altos.

En Brasil, a diferencia de lo que se observó para Argentina, todos los niveles educativos revelan prácticamente una tendencia descendente hasta los 2000 , en particular en el nivel bajo, donde la TGF pasa de 6,1 en 1970 a 3,1 en 2010. El nivel medio comienza en 1970 
con una tasa de 2,6 hijos por mujer y cae hasta 2,4 en 1991, manteniéndose aproximadamente constante hasta 2010. El nivel educativo alto, por su lado, ostenta los menores y descendientes niveles de TGF, mostrando en todo el lapso de estudio un valor por debajo del nivel de reemplazo, tendiente hacia un régimen de muy baja fecundidad en 2010 . El modesto aumento de la fecundidad entre 2000 y 2010 para los grupos de nivel educativo medio y alto es un claro efecto de composición que resulta del importante aumento de la escolaridad en esta década.

\section{GRÁFICO 2}

Tasa global de fecundidad, según nivel educativo (escala logarítmica)

Argentina y Brasil - 1970-2010

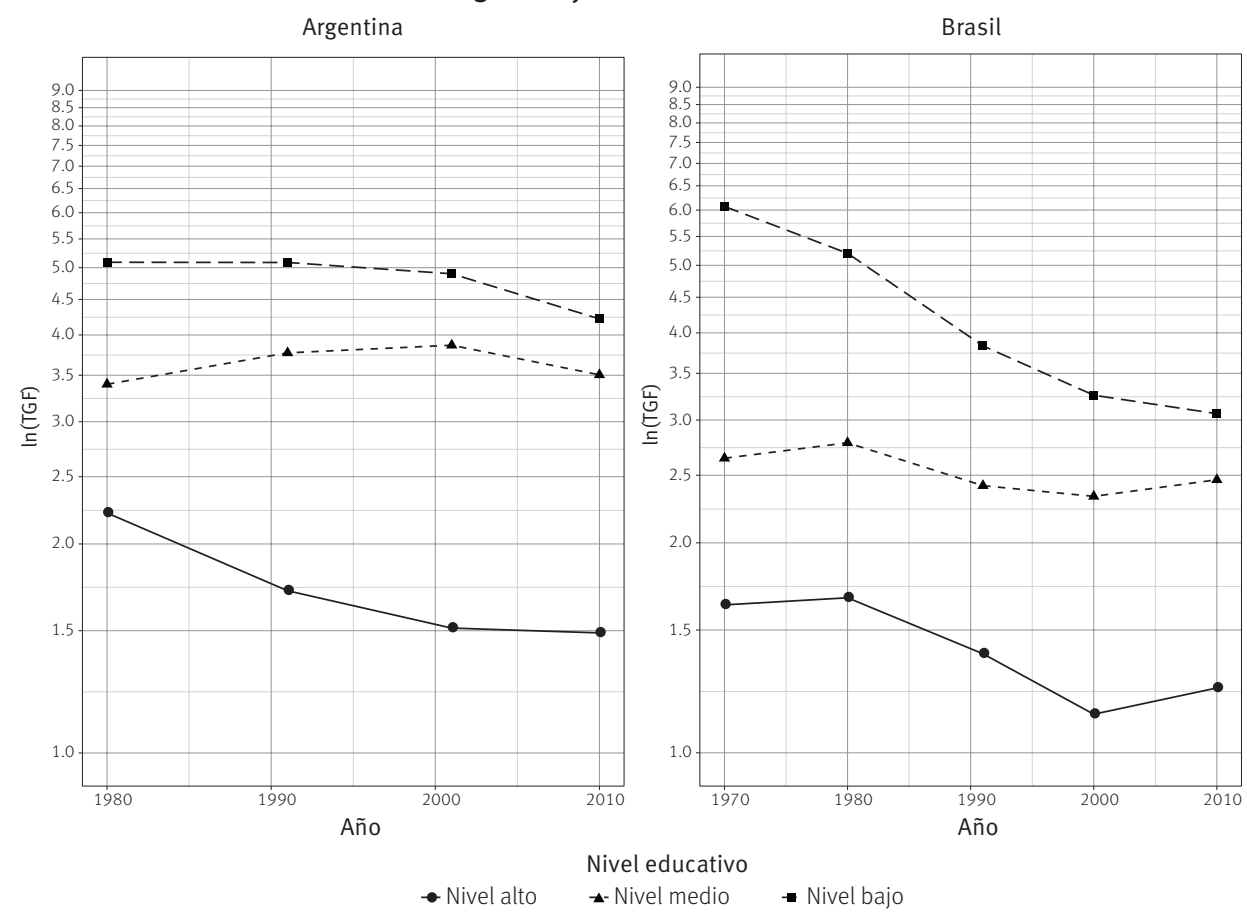

Fuente: Elaboración propia en base a IPUMS-I e INDEC.

Con respecto al universo de mujeres clasificadas según condición socio-ocupacional del jefe/a o persona responsable por el hogar, se distinguen en Argentina (Gráfico 3) niveles descendientes en la TGF para el estrato alto y medio: de 2,8 hijos por mujer en 1980 pasa a 2,1 en 2001. Los empleados revelan una tendencia similar, pero con un nivel de TGF mayor. El pequeño grupo de agricultores presenta los mayores niveles de fecundidad, estables entre 1980 y 1991 (alrededor de 4,4) y descendientes entre 1991-2001. Los trabajadores muestran una TGF descendente en el lapso 1980-1991, aunque con niveles inferiores a los agricultores: pasan de 3,9 hijos por mujer en 1980 a 3,2 en 2001. El estrato bajo, por su lado, manifiesta valores descendentes de la TGF entre 1980-1991 (5,0 y 3,7 respectivamente) estabilizándose alrededor de 3,1 para el 2001. 
En Brasil, todos los grupos socio-ocupaciones, desde 1970 hasta el 2010, ostentan una caída en los niveles de fecundidad. Salvo en 1991, cuando el estrato bajo sobrepasa a los agricultores, ${ }^{7}$ el ranking de los grupos ocupacionales se mantiene a lo largo del periodo de análisis. Ya para el 2010, a excepción de los agricultores, a su vez, todos los grupos muestran una fecundidad por debajo del nivel de reemplazo.

Los datos encontrados para Brasil se muestran consistentes con trabajos previos - por ejemplo (BERQUÓ; CAVENAGHI, 2014; ITABORAÍ, 2015), que, si bien no definen del mismo modo las variables de análisis, se enfocan en la misma problemática.

GRÁFICO 3

Tasa global de fecundidad, según condición socio-ocupacional (escala logarítmica) Argentina y Brasil - 1970-2010
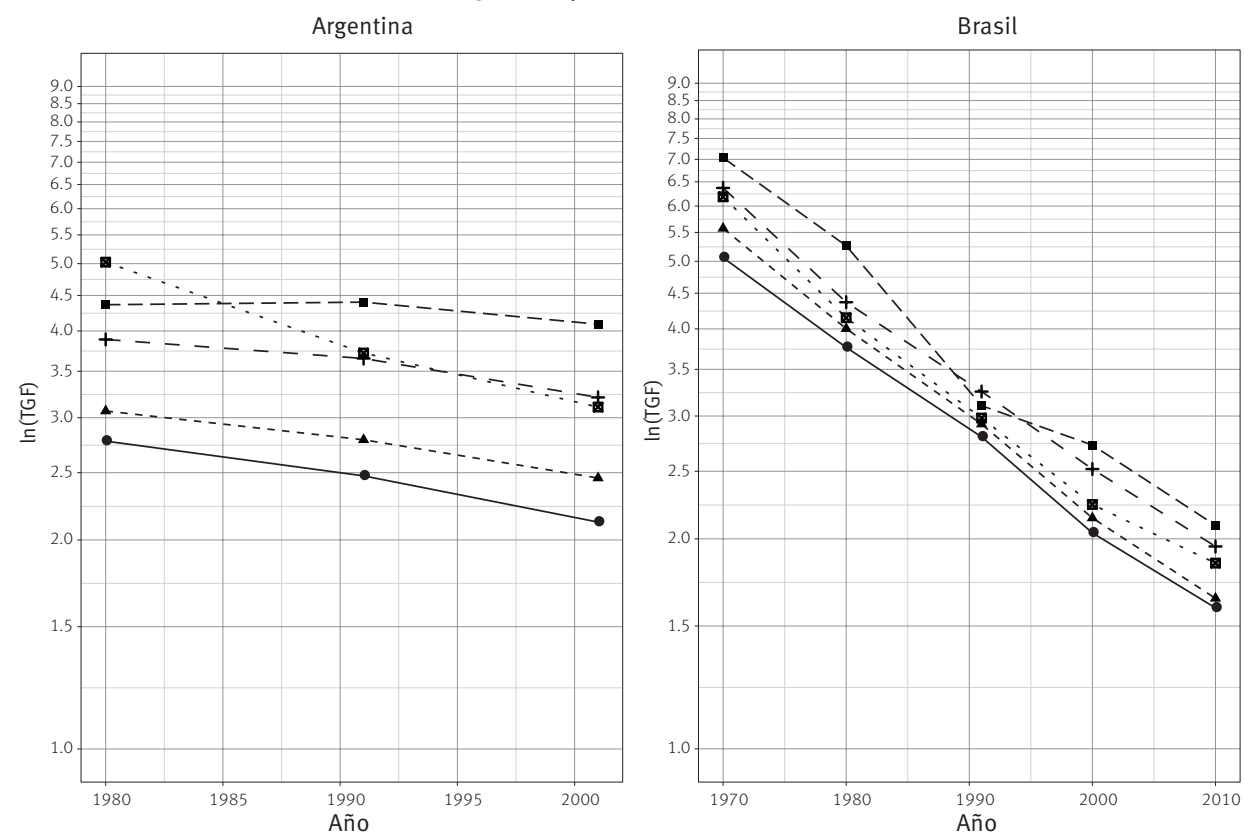

Estrato socio-ocupacional

$\rightarrow$ Alto y medio $\_$-Empleados

- Agricultores + Trabajadores

Fuente: Elaboración propia en base a IPUMS-I e INDEC.

\footnotetext{
${ }_{7}$ Este cambio deberá ser analizado con mayor profundidad a futuro. De forma tentativa se plantea la hipótesis de que sea efecto del fuerte cambio en la composición de la estructura ocupacional de Brasil entre 1980 y 1991 y/o problemas en la calidad de la información de este grupo en el Censo 1991.
} 


\section{Convergencia de la fecundidad de Brasil y Argentina en clave comparativa}

El Gráfico 4 muestra el coeficiente de variación de la TGF por país, año y dimensión analizada, lo que permite evaluar tendencias de convergencia sigma. El coeficiente de variación por regiones en Argentina desciende entre 1980 hasta 1991, cuando se estanca la variabilidad regional. En efecto, para 2001 el CV se mantiene prácticamente en el mismo nivel, pero retoma una tendencia descendente más pronunciada durante el periodo 2001-2010, indicando fuerte convergencia para este último período. En el caso de Brasil, los valores del $C V$ son sistemáticamente descendentes a partir de 1980, con fecundidad convergente, a excepción del período 1970-1980, pero se encuentran, en general, por encima de los de Argentina, lo que refleja las más altas desigualdades regionales.

La dimensión educación es la que presenta la más alta desigualdad en ambos países, aunque con tendencias temporales distintas. El CV de la TGF por nivel educativo en Argentina es ascendente a lo largo de los años 1980-2000, declinando en el lapso 2000-2010. A diferencia de Argentina, en Brasil el CV es tendencialmente descendiente, con valores que fluctúan alrededor de 0,55 en 1970 a 0,34 en 2010 (Gráfico 4).

\section{GRÁFICO 4}

Coeficiente de variación de la TGF, según dimensión analizada Argentina y Brasil - 1970-2010
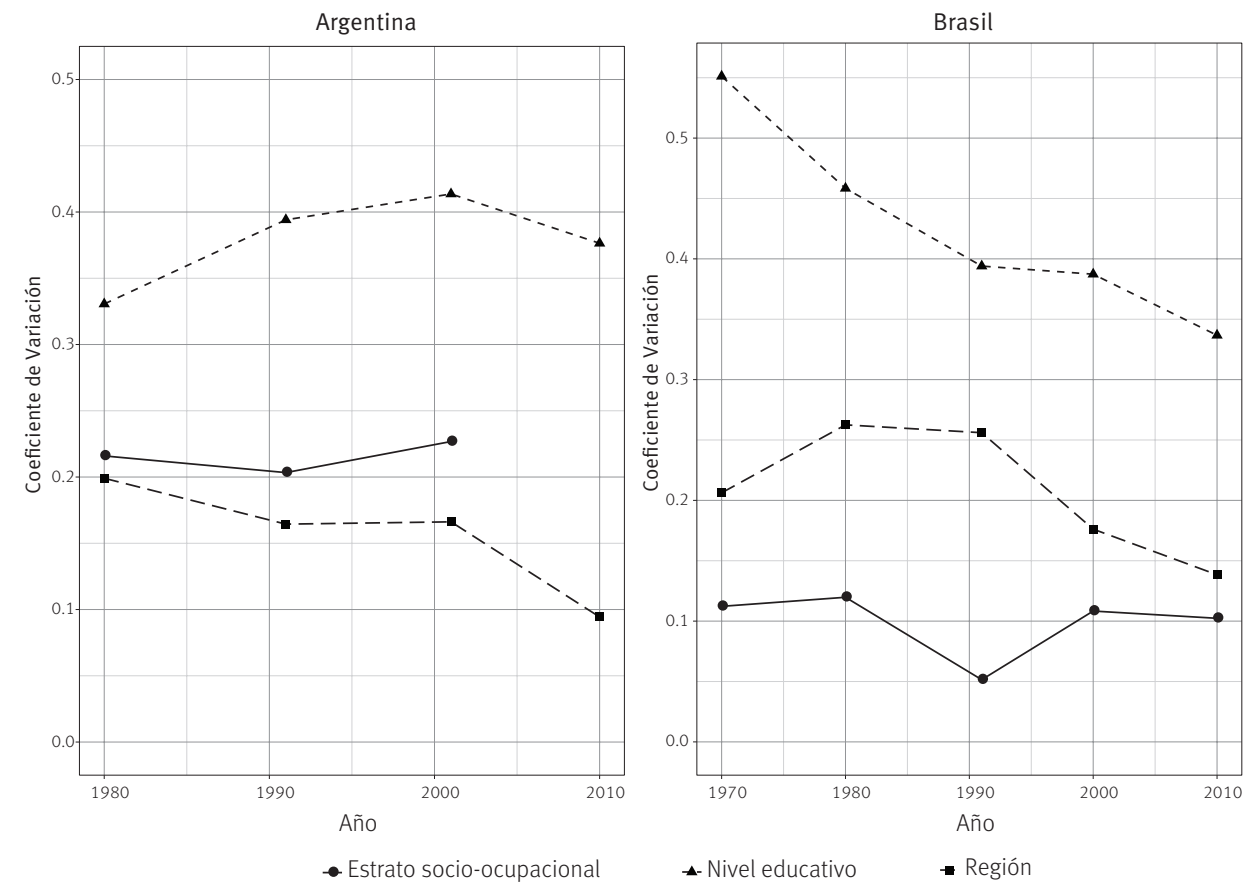

Fuente: Elaboración propia en base a IPUMS-I e INDEC.

La más alta diferencia entre Argentina y Brasil en términos del coeficiente de variación de la TGF es según el estrato socio ocupacional. Los diferenciales de la fecundidad son 
significantemente más altos en Argentina que en Brasil. En Argentina, el CV es levemente descendente a lo largo de 1980-1991 y creciente entre 1991 y 2001. En Brasil, la variación por estrato ocupacional es muy baja $(0,10)$ y aproximadamente constante en todo el periodo de análisis, a excepción de 1991, cuando la variación cae en función de la fuerte caída de la TGF de los agricultores.

Los niveles de convergencia regional considerados a partir del coeficiente $\beta$ (Gráfico 5) en Brasil son divergentes durante 1970-1980 (valores positivos) y altos de convergencia en el resto de los sub-periodos, en especial durante 1991-2000. Si bien se mantiene una tendencia convergente hacia 2010, su intensidad es menor. Argentina muestra convergencia según este indicador para todo el período de análisis, siendo de menor dispersión entre regiones durante el período 1980-1991, tendencia que sigue levemente entre 1991-2001 para luego retomar un valor de convergencia mucho más alto hacia 2010.

\section{GRÁFICO 5}

Convergencia beta de la TGF, según dimensión analizada Argentina y Brasil - 1970-2010
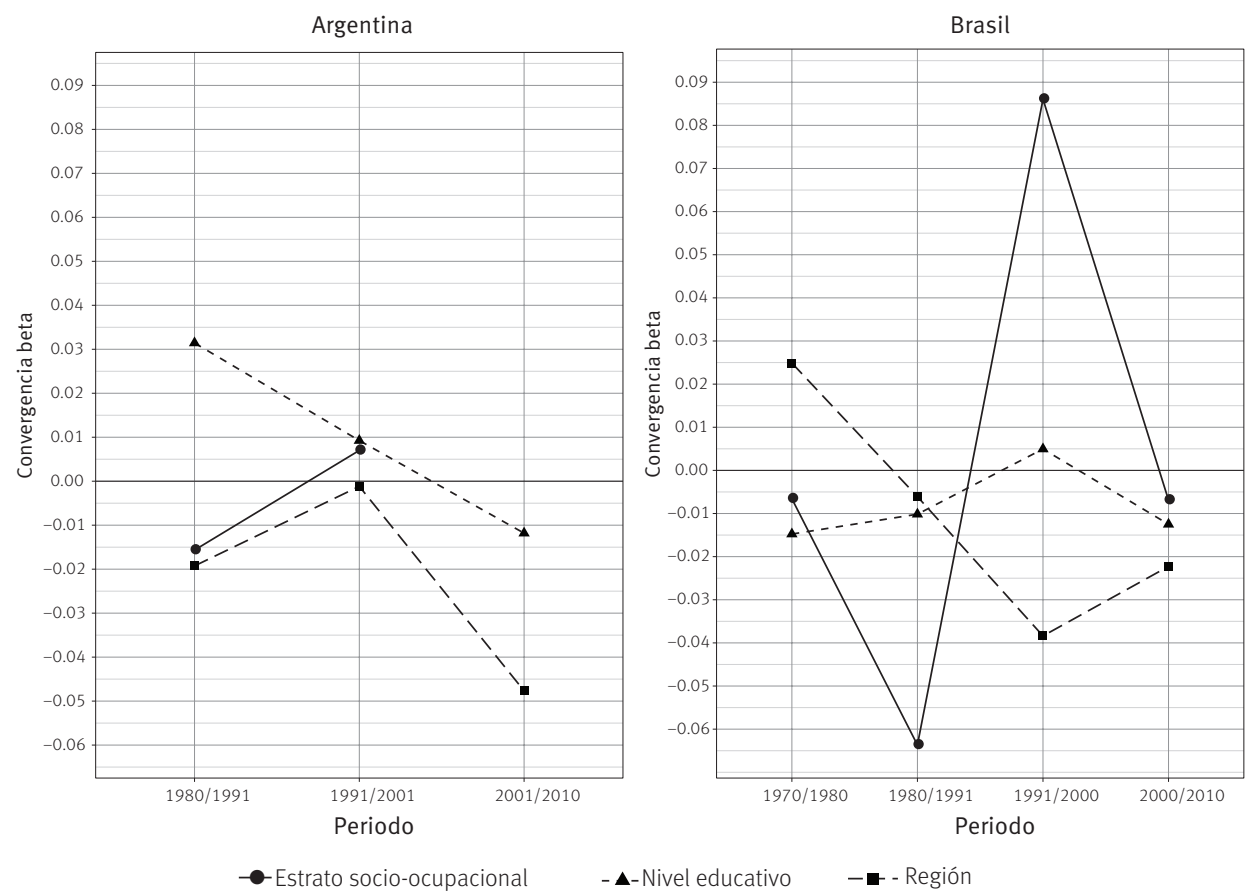

Fuente: Elaboración propia en base a IPUMS-I e INDEC.

En Argentina, los valores de convergencia medidos a partir de $\beta$ para el nivel educativo muestran niveles de divergencia durante 1980-2001 y una convergencia moderada para el período 2001-2010. En Brasil, periodos de convergencia se intercambian con periodos de divergencia según esta variable. Los niveles de convergencia son un poco más altos para el período 1970-1980 (-0.0148), levemente convergentes en 1980-1991 (-0.0102), 
levemente divergentes entre 1991 y 2000 (0.0050) y nuevamente convergentes en 2000 2010 (-0.0125). Los cambios, oscilaciones y niveles observados más que mostrar cambios sugerentes hacia la divergencia/convergencia según niveles educativos sugieren distintos efectos: el gran cambio observado en los niveles educativos de Brasil, en comparación con Argentina, y dentro de cada período intercensal, da cuenta de la heterogeneidad por edad que puede encontrarse al medir la fecundidad según subgrupos educativos. Los efectos de cohorte y del cambio en la distribución de la población de acuerdo a niveles no serían perceptibles de esta forma al medir convergencia según $\beta .{ }^{8}$

En lo que se refiere a los niveles socio-ocupacionales, los valores de convergencia medidos a partir de $\beta$ en Argentina muestran valores de convergencia durante 1980-1991 y niveles divergentes bajos para el período 1991-2001. En Brasil, los valores de $\beta$ expresan convergencia a partir de 1980, a excepción del periodo 1991-2000.

\section{Conclusiones}

Este trabajo ha utilizado informaciones procedentes de los censos demográficos para calcular la fecundidad según distintas variables de diferenciación social para Argentina y Brasil, y testar las hipótesis de convergencia temporal en el promedio de número de hijos de acuerdo a esas dimensiones. A pesar de ser una fuente de dados valiosa y singular, los censos demográficos, y los métodos a los cuales sus datos fueron sometidos, tienen también sus limitaciones. Además de errores derivados de la calidad de la información reportada y el incumplimiento de los supuestos de los métodos, importantes cuestiones interpretativas merecen una atención especial.

Los resultados por niveles educativos, por ejemplo, expresan limitaciones del método aplicado en esta investigación para calcular la fecundidad de acuerdo a la variable P/F, una vez que utiliza información de la fecundidad reciente $(F)$ y la paridez acumulada (P), siendo por eso sensible a cambios estructurales. A su vez, las características de las poblaciones en los dos países han cambiado rápidamente, lo que afecta la interpretación de las tendencias de convergencia según las dimensiones analizadas. A medida que cambia la composición de las cohortes involucradas en el periodo bajo análisis según su nivel educativo, la relación entre educación y fecundidad es más difusa. Otros análisis en profundidad deberían determinar en qué medida estos cambios se deben a los efectos de composición (proporción de personas según nivel) o bien al efecto tasa (cambios en la intensidad de la fecundidad). Para el caso brasilero, se mostró que el efecto de composición explica la mayor parte de la reducción de la fecundidad (RODRÍGUEZ VIGNOLI; CAVENAGHI, 2014).

\footnotetext{
${ }^{8}$ La observación de los niveles absolutos de nivel educativo, en un contexto de expansión de los niveles más altos, pueden no ser la mejor opción para dar cuenta de los diferenciales en fecundidad. En estudio ulteriores, es posible explorar la opción del nivel de educación relativo para compararlo con estos resultados.
} 
A despecho de las limitaciones y cuidados en la interpretación mencionados, los resultados obtenidos permiten llegar a significativas conclusiones. En primer lugar, que la variabilidad inter-regional fue más alta en Brasil para todos los años, incluso con niveles de fecundidad más bajos que en Argentina, desde 1991: durante el lapso 1980-2010 la convergencia a nivel regional de la TGF fue levemente más alta en Brasil que en Argentina. Se observó una divergencia de la TGF entre 1970 y 1980 en Brasil, tendencia que ya se venía expresando en las décadas anteriores en función de la caída de la fecundidad, más acentuada en las regiones más desarrolladas (Sur, Sureste). A la vez, durante el último decenio analizado (2000-2010), la convergencia de la TGF fue mayor entre las regiones de Argentina, cuya variabilidad inter-regional fue menor y el nivel general de la fecundidad más alto que en Brasil.

En segundo lugar, en Brasil las diferencias por región y nivel educativo se mostraron más pronunciadas que las diferencias socio-ocupacionales, aunque más convergentes en el período. En el caso de Argentina, el nivel educativo fue también la dimensión que presentó los más altos diferenciales, pero, a la diferencia de Brasil, las desigualdades socio-ocupacionales en fecundidad son más altas que las regionales. Las tendencias de cierta estabilidad en los diferenciales socio-ocupacionales es una características semejante entre los dos países, mientras que la tendencia general de convergencia de la fecundidad por nivel educativo van en direcciones opuestas.

Retomando la hipótesis de trabajo, al desagregar el análisis de convergencia según región, niveles educativos y categorías socio-ocupacionales, se observó, efectivamente, que la convergencia de la TGF no siempre es verificable, ya que se manifestaron importantes diferenciales de acuerdo a las variables de análisis, aspecto independiente, a la vez, de la etapa de transición de la fecundidad en la que se encuentra cada país durante el periodo de estudio. En definitiva, no hay evidencia de que la fecundidad converja para todas las dimensiones analizadas: la convergencia regional en Brasil comenzó más tardíamente que en Argentina, pero se dio en un contexto de mayor intensidad de la caída de la fecundidad y al mismo tiempo, la convergencia en Argentina comenzó antes, pero su intensidad se vio disminuida durante el decenio 1991-2001.

Además de las diferencias en niveles y velocidad de caída de la fecundidad, en la observación de las tendencias de convergencia según los atributos de análisis, las desigualdades son muy distintas entre los países: al controlar distintas heterogeneidades, se pudo ver, por ejemplo, que en Argentina las desigualdades educativas crecen a lo largo del periodo de estudio, mientras que, en Brasil, la reducción de las brechas según este atributo muestra mayor intensidad.

Por lo visto, estos procesos se han dado de forma distinta entre los dos países analizados; sin embargo, a pesar de estos diferenciales, los resultados llevan a confirmar que la divergencia en las tendencias de la fecundidad ha ocurrido y solo recién hacia el final del periodo de observación son más claros patrones convergentes. Habrá que esperar a ver qué sucede en la dinámica demográfica global y por grupos sociales en estos países, 
en el sentido de si se mantiene o no esta ulterior tendencia hacia la convergencia continua a lo largo del siglo, aspecto que interpela, a su vez, los supuestos que existen por detrás de la mayor parte de las proyecciones de población.

Es posible que el proceso de convergencia en todas las dimensiones observadas en los últimos años persista a futuro. Inicialmente, como consecuencia de un efecto tempo, es posible que la fecundidad en las regiones y grupos sociales más favorecidos deje de caer, o incluso presente leves aumentos, mientras la fecundidad en las regiones y grupos más vulnerables siga cayendo. Esa tendencia puede llevar en el futuro a una nueva etapa de divergencia, con pérdida de la histórica asociación entre características socioeconómicas de las poblaciones y tasas de fecundidad.

Las persistentes desigualdades en relación a necesidades no atendidas en materia de anticoncepción, especialmente entre las mujeres jóvenes, puede llevar al mantenimiento de ciertos diferenciales de fecundidad entre los distintos grupos sociales.

Finalmente, en el análisis comparativo han quedado distinguibles dos fuertes procesos: Argentina, si bien parte de menores niveles que Brasil, solo mantiene una tendencia consistencia de la convergencia de la TGF según la dimensión regional. Se mantienen importantes diferenciales según categoría socio-ocupacional y, principalmente, nivel educativo. En el caso de Brasil, se observa una tendencia de convergencia en el largo plazo para las tres dimensiones, aunque con periodos de divergencia. Se conservan fuertes desigualdades según nivel educativo y reducidos diferenciales de acuerdo a la ocupación.

Quedará pendiente para futuras investigaciones observar la variación conjunta de las variables bajo análisis y observar estas tendencias y diferenciales complementándolas desde el punto de vista de cohorte y con el agregado de un conjunto mayor de países de la región.

\section{Referencias}

ADSERA, A.; MENENDEZ, A. Fertility changes in Latin America in the context of economic uncertainty. Population Studies, n. 65, p. 37-56, 2011.

BAJRAJ, R.; BRAVO, J.; TAPINOS, G. Economic adjustment and demographic responses in Latin America: an overview. In: TAPINOS, G.; MASON, A.; BRAVO, J. (Ed.). Demographic responses to economic adjustment in Latin America. New York: Oxford University Press, 1997.

BERQUÓ, E.; CAVENAGHI, S. Mapeamento sócio-econômico e demográfico dos regimes de fecundidade no Brasil e sua variação entre 1991 e 2000. In: IV ENCONTRO NACIONAL DE ESTUDOS POPULACIONAIS. Anais... Caxambu-MG: Abep, 2004.

Notas sobre os diferenciais educacionais e econômicos da fecundidade no Brasil.

Revista Brasileira de Estudos de População, v. 31, n. 2, p. 471-482, jul./dez. 2014.

BILLARI, F. C. A "great divergence" in fertility? In: POSTON, J. D. (Ed.). Low fertility regimes and demographic and societal change. Cham: Springer, 2018.

BINSTOCK, G.; CERRUTI, M. La población y la estructura social. In: KESSLER, G. (Ed.) La sociedad argentina hoy: radiografía de una nueva estructura social. Buenos Aires: Siglo Veintiuno Editores, 2016. 
BINSTOCK, G.; PANTELIDES, E. A. La fecundidad adolescente hoy: diagnóstico sociodemográfico. In: GOGNA, M. (Ed.). Embarazo y maternidad en la adolescencia. Estereotipos, evidencias y propuestas para políticas públicas. Buenos Aires: Cedes, Ministerio de Salud y Ambiente de la Nación, Unicef Argentina, 2005.

BORGES, G. Theories and measures of demographic convergence: an application for the subnational level in Latin America. In: VII CONGRESO DE LA ASOCIACIÓN LATINOAMERICANA DE POBLACIÓN. Anais... Foz de Iguaçu, Brasil: Alap, 2016.

BRASS, W. Uses of census or survey data for the estimation of vital rates. In: AFRICAN SEMINAR ON VITAL STATISTICS. Anais... Addis Ababa: United Nations Economic Commission for Africa, 1964.

The demography of tropical Africa. Princeton, N.J.: Princeton University Press, 1968.

. Methods for estimating fertility and mortality from limited and defective data. North Carolina: International Program Labs, 1975.

The relational Gompertz model of fertility by age of woman. London: Centre for Population Studies, London School of Hygiene and Tropical Medicine, 1978.

CABELLA, W.; CAVENAGHI, S. Presentación. En: CAVENAGHI, S.; CABELLA, W. (Ed.) Comportamiento reproductivo y fecundidad en América Latina: una agenda inconclusa. Rio de Janeiro: Alap, 2014.

CABELLA, W.; PARDO, I. Hacia un régimen de baja fecundidad en América Latina y el Caribe, 1990-2015. In: CAVENAGHI, S.; CABELLA, W. (Ed.). Comportamiento reproductivo y fecundidad en América Latina: una agenda inconclusa. Rio de Janeiro: Alap, 2014.

. ¿Es hora de usar indicadores refinados para estudiar la fecundidad en América Latina? Revista Brasileira de Estudos de População, v. 33, n. 3, p. 475-493, set./dez. 2016.

CALDWELL, J. C. Demographic theory: a long view. Population and Development Review, v. 30, n. 2, p. 297-316, 2004.

CAMARANO, A. A. Fertility transition in Brazil in the twentieth century: a comparative study of three areas. London: University of London, 1996a.

A hipótese de convergência dos níveis de fecundidade nas projeções populacionais.

Revista São Paulo em Perspectiva, n. 10, p. 18-25, 1996b.

CAMARANO, A. A.; ARAÚJO, H. E.; CARNEIRO, I. G. Tendencias da fecundidade brasileira no século XX: uma visão regional. In: GIFFIN, K.; COSTA, S. H. (Ed.). Questões da saude reprodutiva. Rio de Janeiro: Fiocruz, 1999.

CAMPOS, M.; BORGES, G. Projeção de níveis e padrões de fecundidade no Brasil. In: ERVATTI, L. R.; BORGES, G.; DE PONTE JARDIM, A. (Ed.) Mudança demográfica no Brasil no início do século XXI: subsídios para as projeções da população. Rio de Janeiro: IBGE, 2015.

CANALES, A. I. Retos teóricos de la demográfia en la sociedad contemporánea. Papeles de Población, n. 40, p. 47-69, 2004.

CARVALHO, J. A. Demographic dynamics in Brazil: recent trends and perspectives. Brazilian Journal of Population Studies, n. 1, p. 5-23, 1997.

CARVALHO, J. A.; GONÇALVES, G.; SILVA, L. Aplicação da técnica P/F de Brass em um contexto de rápida queda da fecundidade adolescente: o caso brasileiro na primeira década do século. Belo Horizonte: Cedeplar/UFMG, 2016 (Texto para discussão, n. 540).

CARVALHO, J. A.; WONG, L. R. Fertility transition in Brazil. Causes and consequences. Notas de Población, v. 20, n. 56, p. 107-41, Dec. 1992. 
CASTANHEIRA, H. C.; KOHLER, H.-P. It is lower than you think it is: recent total fertility rates in Brazil and possibly other Latin American countries. Filadélfia, PA: University of Pennsylvania, 2015 (PSC Working Paper Series, n. 63). Disponible en: 〈http://repository.upenn.edu/psc_ working_papers/63〉.

CASTERLINE, J. B. The pace of fertility transition: national patterns in the second half of the twentieth century. Population and Development Review, v. 27 (suppl.), p. 17-52, 2001.

CASTRO, A. F. Social classes and fertility decline in Latin America, 1920-1970. In: POPULATION ASSOCIATION OF AMERICA ANNUAL MEETING. Anais... Chicago: PAA, 2017.

CAVENAGHI, S. Demographic transformations and inequalities in Latin America. In: CAVENAGHI, S. (Ed.). Demographic transformations and inequalities in Latin America: historical trends and recent patterns. Rio de Janeiro: Alap, 2009a.

(Ed). Demographic transformations and inequalities in Latin America: historical trends and recent patterns. Rio de Janeiro: Alap, 2009b.

. Estimaciones y proyecciones de población en América Latina. Desafíos de una agenda pendiente. Rio de Janeiro: Alap, 2012.

CAVENAGHI, S.; ALVES, J. E. D. Diversity of childbearing behaviour in the context of belowreplacement fertility in Brazil. New York: United Nations, Department of Economic and Social Affairs, 2011

. Qualidade das informações sobre fecundidade no Censo Demográfico de 2010. Revista Brasileira de Estudos de População, v. 33, n. 1, p.189-205, jan./abr. 2016.

CELADE - Centro Latinoamericano y Caribeño de Demografía. La nueva era demográfica en América Latina y el Caribe. La hora de la igualdad según el reloj poblacional. In: PRIMERA REUNIÓN DE LA MESA DIRECTIVA DE LA CONFERENCIA REGIONAL SOBRE POBLACIÓN Y DESARROLLO DE AMÉRICA LATINA Y EL CARIBE. Anais... Santiago de Chile: Cepal, 2014. Disponible en: 〈http:// www.cepal.org/celade/noticias/paginas/6/53806/MPD_ddr2_esp.pdf〉.

CEPAL - Comissão Econômica para a América Latina e o Caribe. Panorama social de América Latina 2005. Santiago de Chile: Cepal, 2006. Cepal, 2010.

La hora de la igualdad: brechas por cerrar, caminos por abrir. Santiago de Chile:

Panorama social de América Latina. Santiago de Chile: Cepal, 2011.

Pactos para la igualdad: hacia un futuro sostenible. Santiago de Chile: Cepal, 2014.

CHACKIEL, J. La dinamica demografica en América Latina. Santiago de Chile: Celade, Naciones Unidas, $2004 a$.

La transición de la fecundidad en América Latina 1950-2000. Papeles de Población, n. 10, p. 9-58, 2004 b.

CHACKIEL, J.; SCHKOLNIK, S. Los sectores rezagados en la transición de la fecundidad en América Latina. Revista de la Cepal, n. 83, p. 13-31, 2004.

CHESNAIS, J. C. The demographic transition: stages, patterns, and economic implications: a longitudinal study of sixty-seven countries covering the period 1720-1984. Oxford (England): Clarendon Press; New York: Oxford University Press, 1992.

COALE, A. J. La transición demográfica. Santiago de Chile: Centro Latinoamericano de Demografía, 1977. 
COLEMAN, D. A. Populations of the industrial world - a convergent demographic community? International Journal of Population Geography, v. 8, n. 5, p. 319-44, 2002.

DAVIS, K. The Theory of Change and Response in Modern Demographic History. Population Index, v. 29, n. 4, 345-66, 1963.

DE MELLO MOREIRA, M.; FUSCO, W. Filho único no Brasil. In: XX ENCONTRO NACIONAL DE ESTUDOS POPULACIONAIS. Anais... Foz de Iguaçu: Abep, 2016.

DE MIRANDA-RIBEIRO, A.; RIOS-NETO, E.; GARCIA, R. A. Anticipación y postergación de los nacimientos en la transición de la fecundidad en el Brasil. Notas de Población, n. 103, p. 2943, 2016.

DE MIRANDA-RIBEIRO, A.; RIOS-NETO, E.; ORTEGA, J. A. Declínio da fecundidade no Brasil e México e o nível de reposição: efeitos tempo, parturição e quantum. In: RODRIGUEZ WONG, L. L. (Ed.). Población y salud sexual y reproductiva en América Latina. Rio de Janeiro: Alap, 2008.

DI CESARE, M. Patrones emergentes en la fecundidad y la salud reproductiva y sus vínculos con la pobreza en América Latina y el Caribe. Santiago de Chile: Cepal/Celade (Serie Población y Desarrollo, n. 72).

DINIZ ALVES, J. E. A baixa fecundidade brasileira e seus impactos nas projeções populacionais. 2008. Disponible en: 〈http://www.ie.ufrj.br/aparte/pdfs/artigo_pnds_e_projecoes_09jul08. pdf>.

DORIUS, S. F. Global demographic convergence? A reconsideration of changing intercountry inequality in fertility. Population and Development Review, v. 34, n. 3, p. 519-37, 2008.

DYSON, T. Population and development: the demographic transition. London; New York: Zed Books, 2010.

FARIA, V. Políticas de governo e regulação da fecundidade: conseqüências não antecipadas e efeitos perversos. São Paulo: Cebrap, 1989.

FERNANDEZ, R. E.; CARVALHO, J. A. The evolution of fertility in Brazil, 1957-1979. The application of the own children method to estimate fertility from year to year. Revista Brasileira de Estudos de População, v. 3, n. 2, p. 67-86, 1986.

FRIAS, L. A.; OLIVEIRA, J. D. Fertility levels, trends, and differentials in Brazil since the 1930s. Revista Brasileira de Estudos de População, v. 8, p. 72-111, 1991.

GIUSTI, A. Finalizó la transición de la fecundidad en Argentina? In: IV CONFERENCIA LATINOAMERICANA DE POBLACIÓN. La transición demográfica en América latina y el Caribe. Anais... México: Inegi-Usanam, 1998.

GOLDANI, A. M. Rethinking Brazilian fertility decline. In: XXIV GENERAL POPULATION CONFERENCE INTERNATIONAL UNION FOR THE SCIENTIFIC STUDY OF POPULATION. Anais... Salvador, Bahia: IUSSP-UIESP, 2001.

What will happen to Brazilian fertility? Completing the Fertility Transition. Population Bulletin of the United Nations, n. 48/49, 2002.

GOVEA BASCH, J. E. El estancamiento del descenso de la fecundidad en países de fecundidad intermedia. Evidencias del caso argentino. México: El Colegio de México, Centro de Estudios Demográficos, Urbanos y Ambientales,2013.

GRUSHKA, C. O. Panorama demográfico en Argentina. In: GRAGNOLATI, M.; ROFMAN, R.; APELLA, I.; TROIANO, S. (Ed.). Los años no vienen solos: oportunidades y desafios economicos de la transicion demografica en Argentina. Banco Mundial, 2014. 
GUZMÁN, J. M. The onset of fertility decline in Latin America. Course of Fertility Transition in SubSaharan Africa. Harare, Zimbabwe: International Union for the Scientific Study of Population, 1991.

GUZMÁN, J. M.; RODRÍGUEZ, J.; MARTíNEZ, J.; CONTRERAS, J. M.; GONZÁLEZ, D. The demography of Latin America and the Caribbean since 1950. Population-E, n. 61, p. 519-76, 2005.

GUZMÁN, J. M.; SINGH, S.; RODRIGUEZ, G.; PANTELIDES, E. A. The fertility transition in Latin America. Oxford: Oxford University Press, Clarendon Press, 1996.

IBGE. Projeções da população: Brasil e Unidades da Federação. Rio de Janeiro: IBGE, 2013 (Série Relatórios Metodológicos).

INDEC. Proyecciones provinciales de población por sexo y grupo de edad 2010-2040. Buenos Aires: Indec, 2013 (Serie Análisis Demográfico, n. 35).

ITABORAÍ, N. R. Las desigualdades de clase en el comportamiento reproductivo en el Brasil: democratización incompleta y paradojas de la fecundidad juvenil. Notas de Población, v. 42, n. 100, p. 61-89, 2015.

A maternidade adiada e reduzida e a infecundidade no Brasil: transformações de gênero e desigualdades de classe. In: I CONGRESO DE LA ASOCIACIÓN LATINOAMERICANA DE POBLACIÓN E XX ENCONTRO NACIONAL DE ESTUDOS POPULACIONAIS. Anais... Foz do Iguaçu: Alap/Abep, 2016.

JOHNSON-HANKS, J. Demographic transitions and modernity. Annual Review of Anthropology, v. 37, p. 301-15, 2008.

JONES, G. W. The continuing demographic transition. Oxford; New York: Clarendon Press; Oxford University Press, 1997.

KENT, M. M.; HAUB, C. Global demographic divide. Population Reference Bureau, n. 60, 2005.

KIRK, D. Demographic transition theory. Population Studies, v. 50, p. 361-87, 1996.

KOHLER, H.-P.; ANDERSON, T. Divergent demographic destinies. In: POPULATION ASSOCIATION OF AMERICA ANNUAL MEETING. Anais... Washington, D.C.: PAA, 2016.

LANDRY, A. Adolphe Landry on the demographic revolution. Population and Development Review, v. 13, n. 4, p. 731-40, 1987.

LANZIERI, G. Is there a fertility convergence across member states of the European union? In: JOINT EUROSTAT-UNECE WORK SESSION ON DEMOGRAPHIC PROJECTIONS. Anais... Lisboa: United Nations Statistical Commission and Economic Commission for Europe, 2010.

LEE, R. The demographic transition: three centuries of fundamental change. Journal of Economic Perspectives, v. 17, n. 4, p. 167-90, 2003.

LEE, R.; REHER, D. S. The landscape of demographic transition and its aftermath. Population and Development Review, v. 37 (supplement), p. 1-7, 2011.

LÓPEZ, E. La fecundidad adolescente en la Argentina: desigualdades y desafíos. Encrucijadas UBA, n. 39, p. 24-31, 2006.

LÓPEZ, E.; MARIO, S. La fecundidad en la Argentina 1996-2006: convergencias y divergencias. Población, n. 2, p. 41-58, 2009.

MACINNES, J.; PÉREZ DÍAZ, J. Transformations of the world's population: the demographic revolution. In: TURNER, B. S. (Ed.). The Routledge international handbook of globalization studies. Abingdon, England: Routledge, 2009.

MARTINE, G. Brazil's fertility decline, 1965-95: a fresh look at key factors. Population Development Review, n. 22, p. 47-75, 1996. 
MAZZEO, V. Dinámica demográfica de Argentina en el período 1947-1991. Análisis de sus componentes y diferenciales. In: II JORNADAS ARGENTINAS DE ESTUDIOS DE LA POBLACIÓN. Anais... Aepa, 1995. p. 19-34.

MERRICK, T.; BERQUÓ, E. The determinants of Brazil's recent rapid fertility decline. Washington, D.C.: Report/Committee on Population and Demography, National Academy Press, 1983.

MOULTRIE, T. Evaluation of data on recent fertility from censuses. In: MOULTRIE, T.; DORRINGTON, R.; HILL, A.; HILL, K.; TIMÆUS, I.; ZABA, B. (Ed.). Tools for demographic estimation. Paris: International Union for the Scientific Study of Population, 2013a.

The relational Gompertz model. In: MOULTRIE, T.; DORRINGTON, R.; HILL, A.; HILL, K.; TIMAUS, I.; ZABA, B. (Ed.). Tools for demographic estimation. Paris: International Union for the Scientific Study of Population, 2013b.

MOULTRIE, T.; DORRINGTON, R. E. Sources of error and bias in methods of fertility estimation contingent on the $\mathrm{P} / \mathrm{F}$ ratio in a time of declining fertility and rising mortality. Demographic Research, v. 19, p. 1635-62, 2008.

NATHAN, M. La creciente heterogeneidad en la edad al primer hijo en el Uruguay: un análisis de las cohortes de 1951 a 1990. Notas de Población, n. 100, p. 35-59, 2015.

NATHAN, M.; PARDO, I. Fertility postponement and regional patterns of dispersion in age at first birth. In: VII CONGRESO DE LA ASOCIACIÓN LATINOAMERICANA DE POBLACIÓN. Anais... Foz do Iguaçu: Alap, 2016.

NATHAN, M.; PARDO, I.; CABELLA, W. Diverging patterns of fertility decline in Uruguay. Demographic Research, v. 34, n. 20, p. 563-86, 2016.

NOTESTEIN, F. W. Population: the long view. In: SCHULTZ, T. W. (Ed.). Food for the world. Chicago, Illinois: University of Chicago Press, 1945.

OIT. Sitio web de la CIUO [Online]. Organización Internacional del Trabajo. 2010. Disponible en: 〈http://www.ilo.org/public/spanish/bureau/stat/isco/〉. Consultado en: 1 fev. 2016.

ONU. Completing the fertility transition. New York: Department of Economic and Social Affairs, United Nations, 2002.

World population prospects: the 2017 revision. Methodology of the United Nations Population Estimates and Projections. New York: Department of Economic and Social Affairs, Population Division, 2017.

OTERO, H. La transición demográfica argentina a debate. Una perspectiva espacial de las explicaciones ideacionales, económicas y político-institucionales. In: OTERO, H. (Ed.). El mosaico argentino. Modelos y representaciones del espacio y de la población, siglos XIX-XX. Buenos Aires: Siglo XXI de Argentina Editores, 2004.

PANTELIDES, E. A. La fecundidad argentina desde mediados del siglo XX. Buenos Aires: Cenep, 1989.

. Diferenciales de fecundidad en la Transición Demográfica. In: OTERO, H.; VELÁZQUEZ, G. (Ed.). Poblaciones Argentinas. Estudios de demografía diferencial. Propiep (IEHS-CIG), 1997.

. La transición de la fecundidad en la Argentina 1869-1947. Buenos Aires: Cenep, 2006.

PARRADO, E. A.; MORGAN, S. P. Intergenerational fertility among hispanic women: new evidence of immigrant assimilation. Demography, v. 45, n. 3, p. 651-71, 2008.

PATARRA, N. Transição demográfica: novas evidências, velhos desafios. Revista Brasileira de Estudos de População, v. 11, n. 1, p. 27-40, 1994. 
POTTER, J. E.; SCHMERTMANN, C. P.; ASSUNÇÃO, R. M.; CAVENAGHI, S. M. Mapping the timing, pace, and scale of the fertility transition in Brazil. Population and Development Review, v. 36, n. 2, p. 283-307, 2010.

REHER, D. S. Back to the basics: mortality and fertility interactions during the demographic transition. Continuity and Change, v. 14, n. 1, p. 9-31, 1999.

The demographic transition revisited as a global process. Population, Space and Place, v. 10, n. 1, p. 19-41, 2004.

Towards long-term population decline: a discussion of relevant issues. European Journal of Population, v. 23, n. 2, p. 189-207, 2007.

RIOS-NETO, E. Passado, presente e futuro da fecundidade: uma visão de idade, período e coorte. Revista Brasileira de Estudos de População, v. 17, n. 1-2, p. 6-15, 2000.

RIOS-NETO, E. L. G.; DE MEIRELES GUIMARÃES, R. R. The educational gradient of low fertility in Latin America. In: ANNUAL MEETING OF POPULATION ASSOCIATION OFAMERICA. Anais... Boston, MA: PAA, 2014.

RIPSA. Indicadores e dados básicos - Brasil. Rede Interagencial de Informações para a Saúde, 2012. Disponible en: 〈http://tabnet.datasus.gov.br/cgi/idb2012/matriz.htm〉.

RODRÍGUEZ, J. Vulnerabilidad demográfica en América Latina: q qué hay de nuevo? In: SEMINARIO INTERNACIONAL “LAS DIFERENTES EXPRESIONES DE LA VULNERABILIDAD SOCIAL EN AMÉRICA LATINA Y EL CARIBE”. Anais... Santiago de Chile: ONU-Cepal, 2001.

RODRÍGUEZ VIGNOLI, J.; DI CESARE, M.; PÁEZ, K. Reproducción temprana. Diferencias entre grandes regiones del mundo al inicio y al final de la adolescencia. Cepal/Celade, 2017 (Serie Población y Desarrollo).

RODRÍGUEZ VIGNOLI, J.; CAVENAGHI, S. Adolescent and youth fertility and social inequality in Latin America and the Caribbean: what role has education played? Genus, v. 70, n. 1, p. 1-25, 2014.

RODRIGUEZ WONG, L. L. Fertility in Brazil (urban and rural) - application of the own children method to the 1970 census. Inf Demogr, v. 9, p. 53-97, 1983.

ROSERO-BIXBY, L.; CASTRO-MARTÍN, T.; MARTÍN-GARCÍA, T. Is Latin America starting to retreat from early and universal childbearing? Demographic Research, v. 20, p. 169-94, 2009.

SACCO, N. Evaluación general de los datos de fecundidad del Censo 2010. In: XIII CONGRESO DE LA ASOCIACIÓN ARGENTINA DE ESTUDIOS DE POBLACIÓN. Anais... Universidad Nacional de Salta, Asociación Argentina de Estudios de Población, 2015.

SACCO, N.; RIVEIRO, M. La clasificación de ocupaciones en el Sistema Estadístico Nacional. Estudios del Trabajo, n. 51, jun. 2016.

SALA-I-MARTIN, X.; CENTRE FOR ECONOMIC POLICY, R. The classical approach to convergence analysis. London: Centre for Economic Policy Research,1995.

SCHKOLNIK, S.; CHACKIEL, J. Less advanced sectors in the Latin American fertility transition. CEPAL Review, n. 83, p. 13-30, 2004.

SCHMERTMANN, C. P.; CAVENAGHI, S. M.; ASSUNÇÃO, R. M.; POTTER, J. E. Bayes plus Brass: estimating total fertility for many small areas from sparse census data. Population Studies, v. 67, p. 225-73, 2013.

THOMPSON, W. Population. American Journal of Sociology, v. 34, p. 959-75, 1929.

TORRADO, S. Estructura social de la Argentina, 1945-1983. Buenos Aires: Ediciones de la Flor, 1992.

Procreacion en la Argentina. Hechos e ideas. Buenos Aires: Ediciones de la Flor, 1993. 
Historia de la familia en la Argentina moderna (1870-2000). Buenos Aires: Ediciones de la Flor, 2003.

Transición de la fecundidad. Los hijos: ¿cuántos? ¿cuándo? In: TORRADO, S. (Ed.). Población y bienestar en Argentina del primero al segundo centenario. Una historia social del siglo XX. Buenos Aires: EDHASA, 2007.

VALLIN, J.; MESLÉ, F. Convergences and divergences in mortality: a new approach of health transition. Demographic Research, Special Collection 2, p. 11-44, 2004.

WILSON, C. On the scale of global demographic convergence 1950-2000. Population and Development Review, v. 27, n. 1, p. 155-71, 2001.

Thinking about post-transitional demographic regimes: A reflection. Demographic Research, v. 28, p. 1373-88, 2013.

WOOD, C. H.; CARVALHO, J. A. M. D. A demografia da desigualdade no Brasil. Rio de Janeiro: PNPE/Ipea, 1994.

ZABA, B. Use of the relational Gompertz model in analysing fertility data collected in retrospective surveys. London: Centre for Population Studies, 1981 (CPS Working Paper, n. 81-2).

ZANATTA COUTINHO, R. The transition to low fertility in Brazil. Doctor of Philosophy in Sociology, University of North Carolina at Chapel Hill, Department Program in the College of Arts and Sciences, 2016.

ZAVALA DE COSÍO, M. E. La transición demográfica en América Latina y en Europa. Notas de Población, n. 56, p. 11-32, 1992

. Dos modelos de transicion demografica en America Latina. Perfiles Latinoamericanos: revista de la Sede Academica de México de la Facultad Latinoamericana de Ciencias Sociales, v. 4, p. 29-47, 1995.

\section{Sobre los autores}

Nicolas Sacco es investigador posdoctoral en Demografía en el Centro de Desarrollo y Planificación Regional (Cedeplar), Universidad Federal de Minas Gerais, Investigador Afiliado al Instituto de Investigación en Población y Docente en la Maestría de Demografía Aplicada, Universidad Estatal de Pennsylvania (2017-19). Fue Investigador Estudiante Visitante del Departamento en Demografía, Universidad de California, Berkeley (2015-17). Doctor en Ciencias Sociales (especialización en Demografía Social) por la Universidad de Buenos Aires.

Gabriel Borges es actuario (Universidad Federal de Minas Gerais), magister en Estudios de Población e Investigación Social (Escuela Nacional de Ciencias Estadísticas - Ence) y estudiante de doctorado en Demografía (University of California, Berkeley). Investigador de la Coordinación de Población e Indicadores Sociales del Instituto Brasilero de Geografía y Estadística (IBGE).

\section{Dirección para correspondencia}

Nicolas Sacco

Av. Antônio Carlos, 6627, Oficina 3128, Campus Pampulha

31270-901 - Belo Horizonte-MG, Brasil

Gabriel Borges

Av. Presidente Antônio Carlos, 25/803, Centro

20020-010 - Rio de Janeiro-RJ, Brasil 


\section{Resumo}

Há convergência da fecundidade no Brasil e na Argentina? Um enfoque em desigualdades

A ideia de uma tendência da fecundidade na direção de níveis convergentes na América Latina e Caribe tem uma ampla aceitação, tanto em estudos acadêmicos quanto nos pressupostos das projeções de população dos países da região. Contudo, pouco se sabe sobre os processos de convergência demográfica ao se decompor a fecundidade segundo variáveis de diferenciação social. O objetivo deste artigo foi observar de forma comparada a hipótese de convergência demográfica na Argentina e no Brasil de 1970 a 2010 de acordo com uma análise de período das taxas de fecundidade total segundo regiões estatísticas, níveis educativos e categorias sócio-ocupacionais. Os dados provêm dos censos nacionais de população e as variáveis foram harmonizadas de forma a permitir comparação entre ambos os países. Utilizou-se uma modificação da técnica P/F de Brass e do modelo relacional de Gompertz para estimar os níveis de fecundidade, e medidas de convergência e dispersão para avaliar as variáveis de análise. Os resultados mostram evidência de convergência durante o período de estudo, especialmente para o Brasil, ainda que com alguns ciclos de divergência e diferenças notórias entre os dois países, em particular daqueles derivados da posição na estrutura ocupacional.

Palavras-chave: Fecundidade. Convergência demográfica. Estrutura social. Argentina. Brasil.

\section{Abstract}

Does fertility in Brazil and Argentina converge? A focus on inequalities

The idea of trend in fertility levels towards convergence in Latin America and the Caribbean is widely accepted, both in academic studies and in the underlying assumptions of the population projections in the region. However, little is known about the demographic convergence processes when fertility is decomposed according to variables of social differentiation. The aim of this article is to compare the convergence hypothesis in Argentina and Brazil from 1970 to 2010 through the analysis of the evolution of the Total Fertility Rate by statistical regions, educational levels and socio-occupational categories. Data come from national population censuses and variables were harmonized to allow comparisons between both countries. A variation of the Brass $\mathrm{P} / \mathrm{F}$ ratio method and the Gompertz relational model was used to estimate fertility levels and measures of convergence was calculated to assess the hypothesis of convergence according to the categories of analysis. The results show evidence of convergence in fertility during the period of study, especially in Brazil, although some cycles of divergence and marked differences between the two countries were observed, particularly those arising from the position in the occupational structure.

Keywords: Fertility. Demographic convergence. Social structure. Argentine. Brazil. 